\title{
The Normality Domain of a Family of Holomorphic Functions on a Stein Space (II)
}

\author{
Yukinobu Adachi ${ }^{1}$ \\ 1 2-12-29 Kurakuen, Nishinomiya, Hyogo 662-0082, Japan. E-mail: fwjh5864@ nifty.com
}

Received: September 15, 2014 Accepted: October 21, 2014 Online Published: November 5, 2014

doi:10.5539/jmr.v6n4p99

URL: http://dx.doi.org/10.5539/jmr.v6n4p99

\begin{abstract}
Let $X$ be a Stein space. We prove that for any family $\mathcal{F} \subset O(X)$ every normality domain of it in a weak sence is a meromorphically $O(X)$ - convex domain of $X$.
\end{abstract}

Keywords: normality domain on a Stein space in a weak sense, meromorphically convex

\section{Introduction}

Let $X$ be a Stein space (for its definition, see for example Nishino (2001, p. 280) or Gunning and Rossi (2009, p. 209)). For any family $\mathcal{F} \subset O(X)$, we consider the normality domain in a weak sense $D$ of $X$, that is, it is the largest domain in which $\mathcal{F}_{\mid D}$ is a normal family in a weak sense (see Definition 2.1) and we shall prove in Theorem 4.1 that the domain $D$ is meromorphically $O(X)$ - convex (for its definition, see Definition 3.1) and Stein in Theorem 4.4. We use the meaning that a domain $D$ is the largest one if any domain $D^{\prime} \supsetneqq D$ does not satisfy the property of $D$ no longer. In the previous paper Adachi (2013) we proved that normality domain in a strong sense $D$ of a Stein space $X$ is $O(X)$ - convex (as for a normal family in a strong sense and $O(X)$ - convex domain, see Definition 2.1 and 3.1 respectively).

Julia (1926, p. 68) proved that the normality domains in a weak sense in $\mathbf{C}^{n}$ are pseudoconvex and studied on the inverse problem that such domains are ones of holomorphy or not. And Cartan and Thullen (1932, Satz 12, p. 639) proved that the normality domains in a strong sense are domains of holomorphy and ones in a weak sense are domains of meromorphy for the (Stein) unramified Riemann domain by introducing the concept of holomorphical convexity which is one of the key concepts of the complex analysis of several complex variables.

For the case that $X$ is a Stein manifold, Abe and Furushima $(2000,2005)$ proved that normality open sets in a weak sense are meromorphically $O(X)$ - convex and proved that the inverse is true, that is, meromorphically $O(X)$ - convex open sets are normality open sets in a weak sense of some family of $\mathcal{F} \subset O(X)$. But the normality domain in a weak sense of a family of holomorphic functions on a Stein manifold is not necessary $O(X)$ - convex even for the domain in $X=\mathbf{C}$ (See for example Abe, Furushima and Tsuji (1999, p. 23)). The problem that the normality domain in a weak sense when $X$ is a Stein space which has singular points is meromorphically $O(X)$ - convex has not solved.

In Appendix, we remark that when $X=\mathbf{C}^{n}$, the domain is meromorphically $O(X)$ - convex if and only if it is rationally convex in Oka's sense (1984, p.36) and the normality domain in $\mathbf{C}^{n}$ is if and only if rationally convex. It may be a well known fact for the specialists. When $n=1$ every domain is some normality domain by the classical Runge theorem.

\section{Preliminary (1)}

Following sections, let $X$ be a connected Stein space of dimension $n$ such as $n \geqq 1$ and $F=\left\{f_{j}\right\}_{j=1,2, \cdots}$ where $f_{j} \in O(X)$. Let $p$ be an arbitrary point of $X$ and $U(p)$ be a neighborhood of $X$ such that $\psi_{p}: U(p) \rightarrow V$ is a coordinate homeomorphism of $U(p)$ to $V$ which is an analytic covering, that is, a ramified Riemann domain without relative boundary over a domain $W$ in $\mathbf{C}^{n}$ such as $\pi: V \rightarrow W$ where $\pi$ is a natural projection. We denote $U_{\varepsilon}(p)$ such as $U_{\varepsilon}(p) \subset U(p)$ that $U_{\varepsilon}(p):=\psi_{p}^{-1}\left(V_{\varepsilon}\right)$ where $V_{\varepsilon}$ is an analytic covering over a domain $W_{\varepsilon}\left(\pi \circ \psi_{p}(p)\right)=\left\{(z)=\left(z_{1}, \cdots, z_{n}\right) ;\left\|(z)-\pi \circ \psi_{p}(p)\right\|<\varepsilon\right\}$.

Definition 2.1 We call a family $\mathcal{F} \subset O(X)$ is normal in a weak sense at $p \in X$ when there is a coordinate $U(p)$ and for every sequence $F=\left\{f_{j}\right\}_{j=1,2, \cdots} \subset \mathcal{F}$ we have one of the following: 
(a) There is a subsequence $\left\{f_{j_{\lambda}}\right\}$ which converges uniformly to $f \in O(U(p))$ on $U(p)$;

(b) $\left\{f_{j}\right\}$ diverges compactly on $U(p)$, that is, for every compact set $K \subset U(p)$ and every compact set $L$ in $\mathbf{C}$, there is a positive integer $N$ such that $f_{j}(K) \cap L=\emptyset$ for $j \geqq N$.

We call a family $\mathcal{F} \subset O(X)$ is normal in a strong sense at $p \in X$ when there is a coordinate $U(p)$ such that only the case (a) occurs.

Following, we use the term of "normal" as normal in a weak sense unless otherwise mentioned.

Definition 2.2 We call $\mathcal{F} \subset O(X)$ is normal on a domain $D \subset X$ when for every sequence $F=\left\{f_{j}\right\}_{j=1,2, \cdots} \subset \mathcal{F}$ we have one of the following:

(a) $\left\{f_{j}\right\}$ has a subsequence which converges uniformly to $f \in O(D)$ on every compact set in $D$;

(b) $\left\{f_{j}\right\}$ diverges compactly on $D$.

Following proposition is well known.

Proposition 2.3 A family $\mathcal{F} \subset O(X)$ is normal on a domain $D$ in $X$ if and only if $\mathcal{F}$ is normal at every point $p \in D$.

Definition 2.4 For a point $p \in X$ and a sequence $F=\left\{f_{j}\right\}_{j=1,2, \cdots} \subset O(X)$, we denote the cluster set of $F$ at $p$ such as

$F\left(p: \mathbf{P}^{1}\right)=\bigcap_{\varepsilon>0} \bigcap_{N=1}^{\infty} \overline{\bigcup_{j \geqq N} f_{j}\left(U_{\varepsilon}(p)\right)}$,

where $\overline{\bigcup_{j \geqq N} f_{j}\left(U_{\varepsilon}(p)\right)}$ is the closure of $\bigcup_{j \geqq N} f_{j}\left(U_{\varepsilon}(p)\right)$ in $\mathbf{P}^{1}$.

It is easy to see that $F\left(p: \mathbf{P}^{1}\right)$ is a closed set.

Proposition 2.5 If a sequence $F=\left\{f_{j}\right\}_{j=1,2, \cdots} \subset O(X)$ satisfies $F\left(p: \mathbf{P}^{1}\right) \subset \mathbf{C}-\{\alpha, \beta\}$ with $\alpha \neq \beta$ or $F\left(p: \mathbf{P}^{1}\right)=$ $\{\infty\}$, then $F$ is normal at $p$. When only a former case occurs, $F$ is normal in a strong sense at $p$.

Proof. Since $\mathbf{P}^{1}-\{\alpha, \beta, \infty\}$ is tautly imbedded in $\mathbf{P}^{1}, F$ is normal at $p$ by Kobayashi (1998, Theorem 5.1.11). The last statement is easy to see.

Since $F\left(p: \mathbf{P}^{1}\right)$ is a closed set, following corollary is trivial.

Corollary 2.6 If a sequence $F=\left\{f_{j}\right\}_{j=1,2, \cdots} \subset O(X)$ is not normal at $p$, then $F\left(p: \mathbf{P}^{1}\right)=\mathbf{P}^{1}$.

\section{Preliminary (2)}

Definition 3.1 Let $X$ be a Stein space and $D$ be a domain in $X$. We denote a closed set $\tilde{K}$ such as $\tilde{K}:=\{p \in$ $X ; f(p) \in f(K)$ for every $f \in O(X)\}$ where $K$ is a compact set in $X$. We call that $D$ is a meromorphically $O(X)$ convex domain if for every compact set $K$ in $D, \tilde{K} \cap D$ is compact in $D$.

We denote a closed set $\hat{K}$ such as $\hat{K}:=\left\{p \in X ;|f(p)| \leqq \max _{q \in K}|f(q)|=\max |f(K)|\right.$ for every $\left.f \in O(X)\right\}$ where $K$ is a compact set in $X$. We call $D$ a $O(X)$ - convex domain if for every compact set $K$ in $D, \hat{K} \cap D$ is compact in $D$.

The Definition 2.1 in Adachi (2013) is false but every result in it is correct by a little modifications.

By the definition it is trivial that $\tilde{K} \subset \hat{K}$ and $D$ is a meromorphically $O(X)$ - convex domain if $D$ is a $O(X)$ - convex domain. And it is easy to see the following proposition.

Proposition 3.2 In the above definition we may take $K$ as every compact domain in $D$, that is, a closure of relatively compact domain in $D$, in place of compact set $K$ in $D$.

Following, we take a compact set $K$ in Definition 3.1 such as a compact domain always.

Definition 3.3 We call $\Delta$ a $O(X)$ - meromorphic polyhedron when $\Delta$ is a compact domain in a Stein space $\mathrm{X}$ such as a connected component of $O(X)$ - meromorphic polyhedra $\Delta_{0}:=\left\{p \in X ; \varphi_{j}^{-1}\left(A_{j}\right) \ni p, \varphi_{j} \in O(X), A_{j}\right.$ is a compact domain in $\mathbf{C}$ and $\varphi_{j}(X) \ni A_{j}$ where $\left.j=1, \cdots, k\right\}$ which satisfies $\Delta_{0} \Subset X$.

It is easy to see that $\Delta^{\circ}$ (the interior domain of $\Delta$ above) is meromorphically $O(X)$ - convex.

Proposition 3.4 Let $D$ be a meromorphically $O(X)$ - convex domain in a Stein space $X$ and $K$ be a compact domain in $D$. Then there is a $O(X)$ - meromorphic polyhedron $\Delta$ such that the maximal distance of $\left(p, \partial \tilde{K}_{1}\right)$ for every $p \in \partial \Delta$ is arbitrarily small with $\Delta \ni \tilde{K}_{1}$ where $\tilde{K}_{1}$ is a connected component of $\tilde{K}$.

Proof. As $\tilde{K} \cap D$ is a compact set in $D$, we can take a domain $D_{0} \Subset D$ such that $D_{0} \ni \tilde{K}_{1}$ and $D_{0}$ is near to $\tilde{K}_{1}$. Let $\Sigma$ is the boundary of $D_{0}$ and the distance of $\left(\Sigma, \tilde{K}_{1}\right)=\frac{\delta}{2}$ where $\delta>0$ is a sufficiently small and smaller than the 
minimal distance of $\left(D_{0}, D\right)$.

We may assume that for every point of $q \in \Sigma$ there is a function $f_{q} \in O(X)$ such that $f_{q}(V(q)) \cap f_{q}(K)=\emptyset$ where $V(q)$ is a sufficiently small neighborhood of $q$. As $\Sigma$ is a compact set, there are finite points $\left\{q_{1}, \ldots, q_{v}\right\}$ such as $D \ni V\left(q_{1}\right) \cup \cdots \cup V\left(q_{v}\right) \supset \Sigma$ by Borel-Lebesgue lemma.

Now we set $\Delta_{0}=\left\{p \in X ; A_{j}=f_{q_{j}}(K) \ni f_{q_{j}}(p), j=1, \ldots, v\right\}$ and $\Delta$ is a connected component of $\Delta_{0}$ such as $\Delta \ni \tilde{K}_{1}$.

Proposition 3.5 If $\Delta$ is a $O(X)$ - meromorphic polyhedron, then there is a $O(\Delta)$ - analytic polyhedron $\Delta^{\prime}$ (for its definition, see Nishino (2001, p.270) or Gunning an Rossi (2009, p.216)) such that the maximal distance of ( $p, \partial \Delta)$ for every $p \in \partial \Delta^{\prime}$ is sufficiently small with $\Delta^{\prime} \ni \Delta$.

Proof. Let $\Delta$ be the same as in Definition 3.3. If a point $p \in X$ with $p \notin \Delta$ and near $\partial \Delta$, there is a $\varphi_{j} \in O(X)$ such that $\varphi_{j}(p) \notin A_{j}=\varphi_{j}(\Delta)$. Then there is a neighborhood $V\left(\varphi_{j}(p)\right)$ of $\varphi_{j}(p)$ such as $V\left(\varphi_{j}(p)\right) \cap A_{j}=\emptyset$. We can take a rational function $R(z)$ such as $\left|R\left(\varphi_{j}(p)\right)\right|>\max \left|R\left(\varphi_{j}(\Delta)\right)\right|$ where $R \circ \varphi_{j} \in O(\Delta)$. Then by the standard method such as Proposition 3.4, we can take $\Delta^{\prime}$ as above.

Proposition 3.6 Let $D$ be a limit domain of meromorphically $O(X)$ - convex domain such that $D_{i} \Subset D_{i+1}$ and $\lim _{i \rightarrow \infty} D_{i}=D \subset X$ where $X$ is a Stein space. Then $D$ is a meromorphically $O(X)$ - convex domain.

Proof. Let $K$ be a compact domain in $D$. Then there is a $D_{i}$ such that $K \Subset D_{i}$. By the definition of $\tilde{K}, \tilde{K}$ does not depend on $D_{j}(j \geqq i)$ by the definition. And $\tilde{K}$ is compact in $X, \tilde{K} \cap D_{i}=\tilde{K} \cap D_{j+1}=\tilde{K} \cap D_{j+2}=\cdots \Subset D$ for sufficiently large $j$.

Theorem 3.7 Let $D$ be a meromorphically $O(X)$ - convex domain of a Stein space $X$. Then $D$ is a limit domain of some incresing Stein domains.

Proof. Since the interior domain of $O(X)$ - meromorphic polyhedron is meromorphically $O(X)$ - convex and the interior domain of analytic polyhedron is Stein, statements is easy obtained by Proposition 3.4, 3.5 and 3.6.

Lemma 3.8 Let $K_{1}$ and $K_{2}$ be compact domains of a Stein space $X$ such that $K_{1} \Subset K_{2}$. Then $\tilde{K}_{1} \Subset \tilde{K}_{2} \Subset X$.

Proof. Since $\hat{K}_{2} \Subset X$ and $\tilde{K}_{2} \subset \hat{K}_{2}, \tilde{K}_{2} \Subset X$. Let $p$ be an arbitrary point of $\partial \tilde{K}_{2}$. Since $\tilde{K}_{2}$ is a compact set, $p \in \tilde{K}_{2}$ and $p \in \tilde{K}_{2}{ }^{\prime}$ which is a connected component of $\tilde{K}_{2}$. From Proposition 3.4 there is a $O(X)$ - meromorphic polyhedron $\Delta$ such that $\Delta \ni \tilde{K}_{2}{ }^{\prime}$ where $\Delta$ is near to $\tilde{K}_{2}{ }^{\prime}$ such that other points of connected components of $\tilde{K}_{2}$ are outside of $\Delta$. Let $\left\{p_{i}\right\}_{i=1,2, \ldots} \Subset \Delta-\tilde{K}_{2}^{\prime}$ such that $p_{i} \rightarrow p(i \rightarrow \infty)$. For a point $p_{i}$, there is a function $f_{i} \in O(X)$ such that $f_{i}\left(p_{i}\right) \notin f_{i}\left(K_{2}\right)$. Since $\Delta$ is a compact domain, $\max \left|f_{i}(\Delta)\right| \leqq M_{i}$ with $M_{i}>0$. Then $\max \left|\frac{f_{i}}{M_{i}}(\Delta)\right| \leqq 1$ and $\frac{f_{i}}{M_{i}}\left(p_{i}\right) \notin \frac{f_{i}}{M_{i}}\left(K_{2}\right)$. Since $\left\{\frac{f_{i}}{M_{i}}\right\}_{i=1,2, \ldots .} \subset O(X)$ is a normal family on $\Delta$ and by Vitali's theorem, $\frac{f_{i}}{M_{i}}$ converges to $f \in O(\Delta)$ and $f(p) \notin f\left(K_{2}^{\circ}\right)$.

We consider the normality domain $D$ of $\left\{\frac{f_{i}}{M_{j}}\right\}_{i=1,2, \ldots}$ in a strong sense with $D \supset \Delta$. Then $D$ is $O(X)$ - convex and $(D, X)$ is a Runge pair by Theorem 4.4 in Adachi(2013). Then there is a function $g \in O(X)$ with $g(p) \notin g\left(K_{1}\right)$. Since $p$ is an arbitrary point of $\partial \tilde{K}_{2}, \tilde{K}_{1} \Subset \tilde{K}_{2}$.

Problem 3.9 Let $X$ be a Stein space and $D$ be a meromorphically $O(X)$ - convex domain. Then is D Stein? The examples of Abe, Furushima and Tsuji (1999, p. 23) are Stein but one dimensional.

\section{Conclusion}

Theorem 4.1 Let $D$ be the normality domain in a Stein space $X$ of a family $\mathcal{F} \subset O(X)$. Then $D$ is meromorphically $O(X)$ - convex.

Proof. Let $K$ be a compact domain in $D$. We assume that $D$ is not meromorphically $O(X)$ - convex. Since $\tilde{K}$ is a closed set, a point of $p \in \partial \tilde{K} \cap \partial D$ is a point of $\tilde{K}$. (We consider the point of $\partial D$ such as points of $X$ ).

From the assumption, there is a sequence $F=\left\{f_{j}\right\} \subset \mathcal{F}$ such that $F$ is not normal at $p$ and $F\left(p: \mathbf{P}^{1}\right)=\mathbf{P}^{1}$ by Corollary 2.6. We take a compact domain $K^{\prime} \ni K$ and $K^{\prime} \Subset D$. Then $f_{j}(p)$ is an interior point of $f_{j}\left(K^{\prime}\right)$ because $p \in \tilde{K}$ and $f_{j}(p) \in f_{j}(K)$.

At the first $\left\{f_{j}\right\}$ does not compactly diverge on $D$. Then there is a subsequence $\left\{f_{j_{\lambda}}\right\}$ and $\left\{p_{j_{\lambda}}\right\}$ such that $p_{j_{\lambda}} \rightarrow p$ and $f_{j_{\lambda}}\left(p_{j_{\lambda}}\right) \rightarrow \infty(\lambda \rightarrow \infty)$ by Proposition 2.5. By Lemma 3.8 we may assume that $f_{j_{\lambda}}\left(p_{j_{\lambda}}\right) \in f_{j_{\lambda}}\left(K^{\prime}\right)$. Since there is a subsequence $\left\{f_{j_{\lambda_{\mu}}}\right\}$ converges uniformly to $f \in O(D)$ on every compact set in $D,\left|f_{j_{\lambda_{\mu}}}\left(p_{j_{\lambda_{\mu}}}\right)\right|<M<\infty$. It is a contradiction. 
At the second $\left\{f_{j}\right\}$ compactly diverges on $D$. By Proposition 2.5 it is a contradiction because there is a subsequence $\left\{f_{j_{\lambda}}\right\}$ and $\left\{p_{j_{\lambda}}\right\} \in K^{\prime}$ with $f_{j_{\lambda}}\left(p_{j_{\lambda}}\right) \rightarrow \alpha \neq \infty$.

Therefore $\tilde{K} \cap D \Subset D$ and $D$ is meromorphically $O(X)$ - convex. Because a connected component $\tilde{K}_{1}$ of $\tilde{K}$ is contained in the interior of $D$ or in the interior of $\bar{D}^{c}$.

Problem 4.2 Let $X$ be a meromorphically $O(X)$ - convex space and $D$ be the normality domain of $X$. Then is $D$ meromorphically $O(X)$ - convex ?.

Theorem 4.3 (Abe (2005, Theorem 14)) Let X be a Stein space and $D$ be a domain of $X$. Then the following two conditions are equivalent:

(1) $D$ is meromorphically $O(X)$ - convex;

(2) There exists a family $\mathcal{F} \subset O(X)$ such that $D$ is the normality domain of $\mathcal{F}$.

Theorem 4.4 Let $D$ be the normality domain in a Stein space $X$ of a family $\mathcal{F} \subset O(X)$. Then $D$ is Stein.

Proof. If for every sequence $F=\left\{f_{j}\right\}_{j=1,2, \ldots} \subset \mathcal{F}$ only the case (a) in Definition 2.2 occurs, $D$ is $O(X)$ - convex and then Stein by Theorem 4.1 in Adachi (2013). When for some sequence $F=\left\{f_{j}\right\}_{j=1,2, \ldots} \subset \mathcal{F}$ the case (b) in Definition 2.2 occurs, for every compact domain $K$ in $D$, we consider $\hat{K}_{D}=\{p \in D ;|f(p)| \leqq \max |f(K)|$ for every $f \in O(D)\}$. Since $\left\{\left.f_{j}\right|_{D}\right\} \subset O(D)$ diverges compactly on $D$ and we take a compact domain in $D$ with $K^{\prime} \ni K$ and $\Sigma=\partial K^{\prime}$ such that $\left|f_{j}\left(\partial K^{\prime}\right)\right|>M^{\prime}>0$ for sufficiently large $j$ and $\max \left|f_{j}(K)\right|<M^{\prime}$ by the maximam principle. By the standard method there is an analytic polyhedron $\Delta$ of $D$ such that $\bar{K}_{D} \Subset \Delta \Subset K^{\prime}$ where $\overline{K_{D}}$ is a connected component of $\hat{K}_{D}$ which contains $K$. Let $\Delta^{\circ}$ is the interior domain of $\Delta$. Then $\left(\Delta^{\circ}, D\right)$ is a Runge pair and $\bar{K}_{D}=\hat{K}_{\Delta^{\circ}}=\hat{K}_{D}$. Then $D$ is Stein.

From Theorem 4.1, 4.3 and 4.4 it is easy to see the following theorem.

Theorem 4.5 Let $X$ be a Stein space and $D$ be a meromorphically $O(X)$ - convex domain in $X$. Then $D$ is Stein.

\section{Appendix}

Oka (1941, p.36) introduced the concept of the rationally convex domain $D$ in $\mathbf{C}^{n}$ such as $D$ is $\mathcal{R}\left(\mathbf{C}^{n}\right) \cap O(D)$ convex or $D$ can be approximated from the interior by domains of the above class. There is a domain $D$ such that $D$ is not $\mathcal{R}\left(\mathbf{C}^{n}\right) \cap O(D)$ - convex and rationally convex. For a well known example, let $A=\mathbf{C}^{2}(x, y)-\left\{y-e^{x}=0\right\}$ and $A_{m}=\Delta(m) \times \Delta(m)-\left\{\frac{1}{\left|y-e^{x}\right|} \geqq m\right\}$ where $\Delta(m)$ is a disk in $\mathbf{C}$ with the radius $m$ where $m$ is a sufficiently large integer.

It is easy to see that $A_{m} \rightarrow A(m \rightarrow \infty), A_{m}$ is $\mathcal{R}\left(\mathbf{C}^{2}\right) \cap O(D)$ - convex and $A$ is not $\mathcal{R}\left(\mathbf{C}^{2}\right) \cap O(D)$ - convex because $\mathcal{R}\left(\mathbf{C}^{2}\right) \cap O(D)=\emptyset$.

Proposition 5.1 (Abe (2005), p.265). The domain $D$ in $\mathbf{C}^{n}$ is rationally convex if and only if $D$ is meromorphically $O\left(\mathbf{C}^{n}\right)$ - convex.

Proof. By Storzenberg (1962, p.262) every $\mathcal{R}\left(\mathbf{C}^{n}\right) \cap O(D)$ - convex domain is meromorphically $O\left(\mathbf{C}^{n}\right)$ - convex. From Proposition 3.6 rationally convex domain is meromorphically $O\left(\mathbf{C}^{n}\right)$ - convex.

Inversely from Proposition 3.4 every meromorphically $O\left(\mathbf{C}^{n}\right)$ - convex domain can be approximated from the interior by domains of $O\left(\mathbf{C}^{n}\right)$ - meromorphically polyhedron $\Delta$ and it can be approximated from the interior by some domain of the $O(\Delta)$ - convex domain by Proposition 3.5. Since every holomorphic function of $\Delta$ is approximated uniformly on $\Delta$ on a function of $\mathcal{R}\left(\mathbf{C}^{n}\right) \cap O(D), \Delta^{\circ}$ is rationally convex. Then $O\left(\mathbf{C}^{n}\right)$ - convex domain is rationally convex.

From Theorem 4.3 following proposition is trivial.

Proposition 5.2 The domain $D$ in $\mathbf{C}^{n}$ is rationally convex if and only if $D$ is the normality domain of some family $\mathcal{F} \subset O(D)$.

From the classical Runge theorem it is easy to see the following corollary.

Corollary 5.3 Every domain $D$ in $\mathbf{C}$ is the normality domain of some family $\mathcal{F} \subset O(D)$.

\section{References}

Abe, M. (2005). Meromorphic approximation theorem in a Stein space, Annali di Matematica, 184, 263-274. http://dx.doi.org/10.1007/s10231-004-0115-7 
Abe, M., \& Frushima, M. (2000). On the meromorphic convexity of normality domains in a Stein manifold. Manusucripta Math., 103, 447-453. http://dx.doi.org/10.1007/PL00005862

Abe, M., Furushima, M., \& Tsuji, M. (1999). Equicontinuity domain and disk property. Complex Variables, 39 , 19-25. http://dx.doi.org/10.1080/17476939908815178

Adachi, Y. (2013). The normality domain of a family of holomorphic functions on a Stein space. Far East J. Math. Soc. (FJMS), 77, 137-145.

Cartan, H., \& Thullen, P. (1932). Zur Theorie der Singularitäten der Funktionen mehrerer komplexen Veränderlichen. Regularitäts- und Konvergenzbereiche. Math. Ann., 106, 617-647. http://dx.doi.org/10.1007/BF01455905

Gunning, R., \& Rossi, H. (2009). Analytic functions of several complex variables, AMS Chelsea Publishing.

Julia, G. (1926). Sur les familles de fonctions analytiques de plusieurs variables. Acta Math., 47, 53-115. http://dx.doi.org/10.1007/BF02544108

Kobayashi, S. (1998). Hyperbolic Complex Spaces, Grundlehlen der Mathmatishen Wissenschaften 318, SpringerVerlag, Berlin, Heiderberg. http://dx.doi.org/10.1007/978-3-662-03582-5

Nishino, T. (2001). Function Theory in Several Complex Variables. Translations of Mathmatical Monographs Volume, 193, A.M.S.

Oka, K. (1984). Collected Papers, Translated by R.Narasimhan, Ed. R.Remmert, Springer-Verlag, Berlin, Heidelberg. http://dx.doi.org/10.1007/978-3-642-15083-8

Storzenberg, G. (1962). Polynomially and rationally convex sets. Acta Math., 109, 259-289. http://dx.doi.org/10.1007/BF02391815

\section{Copyrights}

Copyright for this article is retained by the author(s), with first publication rights granted to the journal.

This is an open-access article distributed under the terms and conditions of the Creative Commons Attribution license (http://creativecommons.org/licenses/by/3.0/). 